\title{
LOCALLY FINITE GROUPS WHOSE SUBGROUPS HAVE FINITE NORMAL OSCILLATION
}

\author{
F. DE GIOVANNI ${ }^{\bowtie}$, M. MARTUSCIELLO and C. RAINONE
}

(Received 22 July 2013; accepted 30 August 2013; first published online 20 November 2013)

\begin{abstract}
If $X$ is a subgroup of a group $G$, the cardinal number $\min \left\{\left|X: X_{G}\right|,\left|X^{G}: X\right|\right\}$ is called the normal oscillation of $X$ in $G$. It is proved that if all subgroups of a locally finite group $G$ have finite normal oscillation, then $G$ contains a nilpotent subgroup of finite index.
\end{abstract}

2010 Mathematics subject classification: primary $20 \mathrm{~F} 24$.

Keywords and phrases: locally finite group, normal oscillation.

\section{Introduction}

It is well known that a nonabelian group has only normal subgroups if and only if it is the direct product of a quaternion group of order eight by a periodic abelian group with no elements of order four. More generally, the imposition of generalised normality conditions to all subgroups usually has a strong influence on the structure of a group. A famous result of Neumann [9] shows that in a group $G$ every subgroup $X$ has finite index in its normal closure $X^{G}$ if and only if the commutator subgroup $G^{\prime}$ of $G$ is finite. In the dual situation, it has been proved by Buckley et al. [3] that if $G$ is a locally finite group in which every subgroup $X$ is finite over its core $X_{G}$, then $G$ contains an abelian subgroup of finite index.

The consideration of an infinite extraspecial group shows that a group with finite commutator subgroup need not be abelian-by-finite. On the other hand, it is well known that all finite-by-abelian groups are likewise nilpotent-by-finite, and hence in both theorems quoted above the group $G$ contains a nilpotent subgroup of finite index. The aim of this paper is to prove a similar result for locally finite groups in which every subgroup satisfies one of the two generalised normality conditions considered above.

Let $G$ be a group, and let $X$ be a subgroup of $G$. The normal oscillation of $X$ in $G$ is the cardinal number

$$
\min \left\{\left|X: X_{G}\right|,\left|X^{G}: X\right|\right\} .
$$

This work was partially supported by MIUR_PRIN 2009 (Teoria dei Gruppi e Applicazioni). The first author is a member of GNSAGA (INdAM).

(C) 2013 Australian Mathematical Publishing Association Inc. 0004-9727/2013 \$16.00 
Clearly $X$ is normal in $G$ if and only if it has normal oscillation 1 . Moreover, $X$ has finite normal oscillation in $G$ if and only if either $X$ has finite index in its normal closure $X^{G}$ or it is finite over its core $X_{G}$; in particular, finite subgroups and subgroups of finite index have finite normal oscillation. The main result of the paper is the following theorem.

TheOREM. Let $G$ be a locally finite group in which all subgroups have finite normal oscillation. Then $G$ contains a nilpotent subgroup of finite index.

We mention finally that groups in which every (infinite) subgroup either has finite index in its normal closure or is normal in a subgroup of finite index have been investigated in [7].

Most of our notation is standard and can be found in [10].

\section{Proof of the theorem}

Our first lemma proves that if a subgroup $X$ of a group $G$ has finite normal oscillation, then the factor group $X / X_{G}$ must have finite exponent.

Lemma 2.1. Let $G$ be a group, and let $X$ be a subgroup of $G$ such that the index $\left|X^{G}: X\right|$ is finite. Then the group $X / X_{G}$ has finite exponent.

Proof. Put $\left|X^{G}: X\right|=n$, and let $Y$ be the core of $X$ in $X^{G}$. Then the index $\left|X^{G}: Y\right|$ divides $n$ !, and hence the normal subgroup $\left(X^{G}\right)^{n !}$ of $G$ (which is generated by the set $\left.\left\{h^{n !}: h \in X^{G}\right\}\right)$ is contained in $X$. Therefore $X / X_{G}$ has finite exponent.

Recall that the Baer radical of a group $G$ is the subgroup generated by all abelian subnormal subgroups of $G$, and $G$ is called a Baer group if it coincides with its Baer radical, or equivalently if all finitely generated subgroups of $G$ are subnormal. Of course, it follows from the Hirsch-Plotkin theorem that any Baer group is locally nilpotent.

LeMma 2.2. Let $G$ be a group, and let $X$ be a nilpotent subnormal subgroup of $G$ such that the index $\left|X^{G}: X\right|$ is finite. Then the normal closure $X^{G}$ of $X$ is nilpotent.

Proof. Obviously, the normal closure $X^{G}$ is a Baer group, and in particular all its finitely generated subgroups are subnormal. If $Y$ is the core of $X$ in $X^{G}$, the index $\left|X^{G}: Y\right|$ is finite, and hence $X^{G}=Y E$ for some finitely generated subgroup $E$. Therefore $X^{G}$ is nilpotent by Fitting's theorem.

The following consequence of Lemma 2.2 shows in particular that if $G$ is any locally finite group in which all subgroups have finite normal oscillation, then $G$ contains a hyperabelian subgroup of finite index. Here a group is called hyperabelian if it has an ascending normal series with abelian factors.

Corollary 2.3. Let $G$ be an infinite locally finite group whose subgroups have finite normal oscillation. Then $G$ contains an infinite nilpotent normal subgroup. 
Proof. The group $G$ contains an infinite abelian subgroup $A$ by the famous theorem of Hall-Kulatilaka and Kargapolov (see [10, Part 1, Theorem 3.43]). Of course, we may suppose that $A_{G}$ is finite, so that the index $\left|A^{G}: A\right|$ is finite. Then also the core $B$ of $A$ in $A^{G}$ has finite index in $A^{G}$, and so $B$ is an infinite abelian subnormal subgroup of $G$. Moreover, the subgroup $B_{G}$ is obviously finite, so that the index $\left|B^{G}: B\right|$ must be finite, and hence $B^{G}$ is nilpotent by Lemma 2.2.

Lemma 2.4. Let $G$ be a Baer group whose subgroups have finite normal oscillation. Then $G$ is soluble.

Proof. Let $X$ be any subgroup of $G$. If the index $\left|X: X_{G}\right|$ is finite, we have that $X$ is subnormal in $G$ since $G / X_{G}$ is a Baer group. On the other hand, if $X$ has finite index in its normal closure $X^{G}$, then $X$ is subnormal in $X^{G}$, and so even in $G$, because $G$ is locally nilpotent. Therefore all subgroups of $G$ are subnormal, and hence $G$ is soluble by a relevant theorem of Möhres [8].

If $G$ is any group, the set $C(G)$, consisting of all elements of $G$ having only finitely many conjugates, is a characteristic subgroup, called the $F C$-centre of $G$. The upper $F C$-central series of $G$ is the ascending normal series $\left\{C_{\alpha}(G)\right\}_{\alpha}$, defined by the rules $C_{0}(G)=\{1\}$,

$$
C_{\alpha+1}(G) / C_{\alpha}(G)=C\left(G / C_{\alpha}(G)\right)
$$

for each ordinal $\alpha$ and

$$
C_{\lambda}(G)=\bigcup_{\beta<\lambda} C_{\beta}(G)
$$

if $\lambda$ is a limit ordinal. For the main properties of the upper $F C$-central series of a group, we refer to [10, Part 1, Ch. 4]. The behaviour of the upper $F C$-central series of a group in which all subgroups have finite normal oscillation plays a central role in our considerations, and will be described by the following three results.

Lemma 2.5. Let $G$ be a group in which all subgroups have finite normal oscillation, and let $C$ be the $F C$-centre of $G$. Then every subgroup of $C$ has finite index in its normal closure in $G$, and in particular the commutator subgroup $C^{\prime}$ of $C$ is finite.

Proof. Let $X$ be any subgroup of $C$ such that the index $\left|X: X_{G}\right|$ is finite. Then it follows from Dietzmann's lemma that the group $X^{G} / X_{G}$ is finite (see [10, Part 1, page 45]), and so $X$ has finite index in $X^{G}$. In particular, each subgroup of $C$ has finite index in its normal closure in $C$, and hence $C^{\prime}$ is finite.

LemMa 2.6. Let $G$ be a locally finite p-group in which all subgroups have finite normal oscillation, and let $A$ be an abelian normal subgroup of $G$. If $A$ is a direct product of cyclic subgroups and $C=C(G)$ is the $F C$-centre of $G$, then $A / A \cap C$ is finite.

Proof. Assume for a contradiction that $A / A \cap C$ is infinite. Then there exist in $A$ sequences of elements $\left(a_{n}\right)_{n \in \mathbb{N}}$ and $\left(b_{n}\right)_{n \in \mathbb{N}}$ such that

$$
C<\left\langle C, a_{1}\right\rangle<\left\langle C, a_{1}, b_{1}\right\rangle<\left\langle C, a_{1}, b_{1}, a_{2}\right\rangle<\cdots<\left\langle C, a_{1}, b_{1}, \ldots, a_{n}, b_{n}\right\rangle<\cdots
$$


and

$$
\left\langle a_{1}, b_{1}, a_{2}, b_{2}, \ldots, a_{n}, b_{n}, \ldots\right\rangle=\left\langle a_{1}\right\rangle \times\left\langle b_{1}\right\rangle \times\left\langle a_{2}\right\rangle \times\left\langle b_{2}\right\rangle \times \cdots \times\left\langle a_{n}\right\rangle \times\left\langle b_{n}\right\rangle \times \cdots .
$$

Now consider a partition

$$
\left\{I_{n} \mid n \in \mathbb{N}\right\}
$$

of the set $\mathbb{N}$ of natural numbers, consisting of infinitely many infinite subsets. For all positive integers $k$ and $n$, put

$$
U_{k, n}=\left(\operatorname{Dr}_{h \in I_{k}}\left\langle a_{h}\right\rangle\right) \times\left\langle b_{n}\right\rangle .
$$

Since every $b_{n}$ has infinitely many conjugates in $G$, and

$$
U_{r, n} \cap U_{s, n}=\left\langle b_{n}\right\rangle
$$

if $r \neq s$, it follows that for each $n$ there exists at most a positive integer $m$ such that $U_{m, n}$ has finite index in its normal closure. Thus for each positive integer $n$, we can choose a positive integer $k(n)$ such that the core $V_{n}$ of $U_{k(n), n}$ has finite index in $U_{k(n), n}$ and $k(m) \neq k(n)$ if $m \neq n$. Therefore

$$
U=\left\langle U_{k(n), n} \mid n \in \mathbb{N}\right\rangle=\operatorname{Dr}_{n \in \mathbb{N}} U_{k(n), n} .
$$

Clearly, the factor group $U_{k(n), n} C / C$ is infinite, so that for each $n$ the subgroup $V_{n}$ is not contained in $C$. For every positive integer $n$, let $y_{n}$ be an element of $V_{n}$ having infinitely many conjugates in $G$, and put

$$
V=\underset{n \in \mathbb{N}}{\operatorname{Dr}}\left\langle y_{n}\right\rangle .
$$

Then

$$
V_{n} \cap V=\left\langle y_{n}\right\rangle
$$

so that the conjugates of $y_{n}$ are representatives of infinitely many cosets of $V$, and hence the index $\left|V^{G}: V\right|$ is infinite. It follows also that the core $V_{G}$ of $V$ is contained in the $F C$-centre of $G$, so that the index $\left|V: V_{G}\right|$ must be infinite, and this contradiction completes the proof of the statement.

COROLlary 2.7. Let $G$ be a locally finite p-group in which all subgroups have finite normal oscillation, and let $A$ be an abelian normal subgroup of $G$. Then $A$ is contained in the second $F C$-centre $C_{2}(G)$ of $G$.

Proof. If $a$ is any element of $A$, the normal closure $\langle a\rangle^{G}$ is an abelian group of finite exponent, and hence it is a direct product of cyclic subgroups. Then it follows from Lemma 2.6 that $\langle a\rangle{ }^{G} C_{1}(G) / C_{1}(G)$ is a finite normal subgroup of $G / C_{1}(G)$, and so it is contained in the $F C$-centre of $G / C_{1}(G)$. Therefore $A$ is contained in $C_{2}(G)$.

LemMa 2.8. Let $G$ be a p-group, and let $A$ be an abelian normal subgroup of $G$ such that the index $\left|X^{G}: X\right|$ is finite for every subgroup $X$ of $A$. Then $A$ contains a finite $G$-invariant subgroup $B$ such that the centraliser $C_{G}(A / B)$ has finite index in $G$. 
Proof. Write $A=D \times R$, where $D$ is the largest divisible subgroup of $A$ and $R$ is reduced. Let $P$ be any subgroup of type $p^{\infty}$ of $A$. As the index $\left|P^{G}: P\right|$ is finite, the subgroup $P$ is characteristic in $P^{G}$ and so it is normal in $G$. It follows that all subgroups of $D$ are normal in $G$, and in particular $G / C_{G}(D)$ is finite. Moreover, $R$ has finite index in its normal closure $R^{G}$, so that $R^{G}$ is likewise reduced and hence it contains a finite $G$-invariant subgroup $B$ such that all subgroups of $R^{G} / B$ are normal in $G / B$ (see [4, Lemma 2.9]). Therefore the intersection

$$
C_{G}(D) \cap C_{G}\left(R^{G} / B\right)
$$

has finite index in $G$, and so also the index $\left|G: C_{G}(A / B)\right|$ is finite.

Lemma 2.9. Let $G$ be a soluble p-group whose subgroups have finite normal oscillation. Then $G$ contains a nilpotent subgroup of finite index.

Proof. Assume that the statement is false, and choose a counterexample $G$ with minimal derived length $k$. Let $C$ be the $F C$-centre of $G$. It follows from Lemma 2.5 that every subgroup of $C$ has finite index in its normal closure in $G$, and in particular the commutator subgroup $C^{\prime}$ of $C$ is finite. Application of Lemma 2.8 to the factor group $G / C^{\prime}$ yields that there exists a finite $G$ invariant subgroup $E$ of $C$ such that $C^{\prime} \leq E$ and the centraliser $C_{G}(C / E)$ has finite index in $G$. Since every finite-by-nilpotent $p$-group is obviously nilpotent, also the factor group $G / E$ is a minimal counterexample, and its $F C$-centre is $C / E$. Therefore, replacing $G$ by $G / E$, it can be assumed without loss of generality that the centraliser $C_{G}(C)$ has finite index in $G$.

Consider the last nontrivial term $A=G^{(k-1)}$ of the derived series of $G$. By the minimal choice of $k$, the group $G / A$ contains a subgroup of finite index $N / A$ which is nilpotent. Moreover, $A$ is contained in the second $F C$-centre $C_{2}(G)$ of $G$ by Corollary 2.7, and so every subgroup of $A C / C$ has finite index in its normal closure in $G / C$ by Lemma 2.5. Another application of Lemma 2.8 yields that $A C / C$ contains a finite $G$-invariant subgroup $B / C$ such that the centraliser $C_{G}(A C / B)$ has finite index in $G$. Therefore the intersection

$$
M=N \cap C_{G}(C) \cap C_{G}(B / C) \cap C_{G}(A C / B)
$$

is a nilpotent subgroup of finite index of $G$, and this contradiction completes the proof.

We can now prove the statement of our main theorem in the case of primary locally finite groups.

Lemma 2.10. Let $G$ be a locally finite p-group whose subgroups have finite normal oscillation. Then $G$ contains a nilpotent subgroup of finite index.

Proof. Let $F$ be the Fitting subgroup of $G$, that is the subgroup generated by all nilpotent normal subgroups of $G$. Then $F$ is a Baer group, and hence it is soluble by Lemma 2.4. Assume for a contradiction that the index $|G: F|$ is infinite, so that it follows from Corollary 2.3 that $G / F$ contains an infinite nilpotent normal 
subgroup $N / F$. As $N$ is soluble, Lemma 2.9 yields that it is nilpotent-by-finite. Thus the Fitting subgroup $M$ of $N$ is a nilpotent normal subgroup of $G$ and the index $|N: M|$ is finite. This contradiction shows that $F$ has finite index in $G$, so that $G$ is soluble and hence it is nilpotent-by-finite by Lemma 2.9.

Corollary 2.11. Let $G$ be a locally finite group whose subgroups have finite normal oscillation, and let $P$ be a Sylow subgroup of $G$. Then $G$ contains a nilpotent normal subgroup $N$ such that $P N / N$ is finite.

Proof. The subgroup $P$ is nilpotent-by-finite by Lemma 2.10. If the index $\left|P: P_{G}\right|$ is finite, the Fitting subgroup of $P_{G}$ is a nilpotent normal subgroup of $G$ and it has finite index in $P$. On the other hand, if $P$ has finite index in $P^{G}$, the normal subgroup $P^{G}$ is likewise nilpotent-by-finite; in this case, the Fitting subgroup $N$ of $P^{G}$ is nilpotent and $P N / N$ is finite.

If $G$ is any periodic group, we will denote by $\pi(G)$ the set of all prime numbers $p$ such that $G$ has elements of order $p$. Suppose that $\pi(G)=\omega_{1} \cup \omega_{2}$, where $\omega_{1}$ and $\omega_{2}$ are disjoint subsets; if $U$ is an $\omega_{1}$-subgroup of $G$ and $V$ is an $\omega_{2}$-subgroup of $G$ such that $G=U V$, we shall say that $G=U V$ is an $\left(\omega_{1}, \omega_{2}\right)$-decomposition of $G$. Moreover, for each set $\omega$ of prime numbers, $\omega^{\prime}$ will denote the set of primes which are not in $\omega$. Thus the classical result of P. Hall on Sylow theory of finite soluble groups just says that each finite soluble group has an $\left(\omega, \omega^{\prime}\right)$-decomposition for each set $\omega$ of prime numbers. This was extended by E. Schenkman (in an unpublished work) to the case of countable locally finite groups. We give here an easy proof of this latter result.

LemMa 2.12. Let $G$ be a countable periodic locally soluble group, and let $\omega$ be any subset of $\pi(G)$. Then $G=U V$, where $U$ is an $\omega$-subgroup and $V$ is an $\omega^{\prime}$-subgroup of $G$.

Proof. Since $G$ is a countable locally finite group, there exists an ascending chain

$$
\{1\}=G_{0}<G_{1}<G_{2}<\cdots<G_{n}<\cdots
$$

of finite subgroups of $G$ such that

$$
G=\bigcup_{n \in \mathbb{N}_{0}} G_{n} .
$$

Put $U_{0}=V_{0}=\{1\}$, and suppose that for some $n \geq 0$ an $\left(\omega, \omega^{\prime}\right)$-decomposition

$$
G_{n}=U_{n} V_{n}
$$

of $G_{n}$ has been chosen. Now consider any $\left(\omega, \omega^{\prime}\right)$-decomposition

$$
G_{n+1}=A_{n+1} B_{n+1}
$$

of $G_{n+1}$. By Hall's theorem there exist $x$ and $y$ in $G_{n+1}$ such that $U_{n} \leq A_{n+1}^{x}$ and $V_{n} \leq B_{n+1}^{y}$. Moreover,

$$
G_{n+1}=U_{n+1} V_{n+1},
$$


where $U_{n+1}=A_{n+1}^{x}$ and $V_{n+1}=B_{n+1}^{y}$ (see for instance [1, Lemma 1.3.1]). In this way we have defined by induction two ascending chains

$$
\{1\}=U_{0}<U_{1}<\cdots<U_{n}<\cdots
$$

and

$$
\{1\}=V_{0}<V_{1}<\cdots<V_{n}<\cdots
$$

of finite $\omega$-subgroups and finite $\omega^{\prime}$-subgroups of $G$, respectively. Write

$$
U=\bigcup_{n \in \mathbb{N}_{0}} U_{n}
$$

and

$$
V=\bigcup_{n \in \mathbb{N}_{0}} V_{n} .
$$

Therefore $U$ is an $\omega$-subgroup and $V$ is an $\omega^{\prime}$-subgroup, so that $G=U V$ is an $\left(\omega, \omega^{\prime}\right)$ decomposition of $G$ and the statement is proved.

Lemma 2.13. Let $G$ be a countable locally finite group whose subgroups have finite normal oscillation. Then $G$ has a unique Sylow p-subgroup for all but finitely many prime numbers $p$.

Proof. It follows from Corollary 2.3 that every infinite homomorphic image of $G$ has an infinite nilpotent normal subgroup. Then $G$ contains a hyperabelian normal subgroup $H$ of finite index, and it is obviously enough to prove that the statement holds for $H$. Therefore it can be assumed without loss of generality that $G$ itself is hyperabelian.

Let $\omega$ be the set of all prime numbers $p$ such that $G$ contains a nonnormal Sylow $p$-subgroup $G_{p}$, and assume for a contradiction that $\omega$ is infinite. Consider an infinite subset $\omega_{1}$ of $\omega$ such that $\omega \backslash \omega_{1}$ is infinite, and put $\omega_{2}=\pi(G) \backslash \omega_{1}$. As $G$ is a countable locally (soluble and finite) group, it follows from Lemma 2.12 that $G$ has an $\left(\omega_{1}, \omega_{2}\right)$ decomposition $G=U V$. The factor groups $U / U_{G}$ and $V / V_{G}$ have finite exponent by Lemma 2.1, and hence there exist prime numbers $p$ in $\omega_{1}$ and $q$ in $\omega \backslash \omega_{1}$ such that $\left[G_{p}, G_{q}\right]=\{1\}$. An application of Ramsey's theorem yields now that $\omega$ contains an infinite subset $\omega^{*}$ such that

$$
\left[G_{p}, G_{q}\right]=\{1\}
$$

for all distinct primes $p$ and $q$ in $\omega^{*}$. Put

$$
K=\left\langle G_{p} \mid p \in \omega^{*}\right\rangle=\operatorname{Dr}_{p \in \omega^{*}} G_{p} .
$$

As the factor group $K / K_{G}$ has finite exponent, there exists a prime number $q$ in $\omega^{*}$ such that $G_{q}$ is contained in $K_{G}$. It follows that $G_{q}$ is a normal subgroup of $G$, and this contradiction proves that the set $\omega$ is finite. 
Recall that a group class $\mathfrak{X}$ is said to be countably recognisable if it contains all groups whose countable subgroups belong to $\mathfrak{X}$. It is well known that many relevant classes of groups are countably recognisable; in particular, Baer [2] proved that this is true for the class of nilpotent groups. As a consequence of results by Dixon et al. [6], we can prove here that the class of nilpotent-by-finite groups is also countably recognisable. This result of independent interest will be used in the proof of our main theorem, in order to restrict the attention to the case of countable groups.

LeMma 2.14. The class of nilpotent-by-finite groups is countably recognisable.

Proof. Let $G$ be a group whose countable subgroups are nilpotent-by-finite, and let $F$ be the Fitting subgroup of $G$. Then all countable subgroups of $F$ are nilpotent, and hence $F$ itself is nilpotent. Assume for a contradiction that $G / F$ is infinite, and let $H / F$ be a countably infinite subgroup of $G / F$. Then $G$ contains a countable subgroup $X$ such that $H \leq X F$ and the set $X \backslash F$ has empty intersection with the Fitting subgroup $V$ of $X$ (see [6, Corollary 2.3]). Let $h$ and $k$ be elements of $H$. Then $h=x a$ and $k=y b$, where $x, y$ belong to $X$ and $a, b$ are elements of $F$. In particular, if the cosets $h F$ and $k F$ are different, we have also that $x V \neq y V$, since

$$
(X \backslash F) \cap V=\emptyset .
$$

It follows that the factor group $X / V$ is also infinite. This contradiction proves that $G / F$ is finite, and so $G$ is nilpotent-by-finite.

Proof of The Theorem. By Lemma 2.14, it is enough to prove the statement in the case of a countable group $G$. Let $\omega$ be the set of all prime numbers $p$ such that $G$ contains a nonnormal Sylow $p$-subgroup $G_{p}$. Then $\omega$ is finite by Lemma 2.13. Put $\pi=\pi(G) \backslash \omega$, and for each prime $q$ in $\pi$ let $G_{q}$ be the unique Sylow $q$-subgroup of $G$. Consider in $G$ the normal subgroup

$$
K=\operatorname{Dr}_{q \in \pi} G_{q}
$$

Since $G$ is countable and $\pi(K) \cap \pi(G / K)=\emptyset$, it is well known that there exists a subgroup $H$ of $G$ such that $G=H K$ and $H \cap K=\{1\}$ (see for instance [5, Theorem 3.4.5]). Let $\pi^{*}$ be the subset of $\pi$, consisting of all primes $q \in \pi$ such that $G_{q}$ contains a nonnormal subgroup $X_{q}$, and put

$$
X=\operatorname{Dr}_{q \in \pi^{*}} X_{q}
$$

The factor group $X / X_{G}$ has finite exponent by Lemma 2.1, so that the set $\pi^{*}$ must be finite, and hence the subgroup $G_{q}$ is abelian for all but finitely many primes $q$ in $\pi$. On the other hand, it follows from Lemma 2.10 that every Sylow subgroup of $G$ is nilpotent-by-finite, and so the Fitting subgroup $L$ of $K$ is nilpotent and the index $|K: L|$ is finite. As $H \simeq G / K$, the set of primes $\pi(H)$ is finite, and so by Corollary 2.11 there exists a nilpotent normal subgroup $N$ of $G$ such that all Sylow subgroups of $H N / N$ are finite. Therefore $H N / N$ is finite, and hence the nilpotent normal subgroup $L N$ has finite index in $G$. The theorem is proved. 


\section{References}

[1] B. Amberg, S. Franciosi and F. de Giovanni, Products of Groups (Clarendon Press, Oxford, 1992).

[2] R. Baer, 'Abzählbar gruppentheoretische Eigenschaften', Math. Z. 79 (1962), 344-363.

[3] J. Buckley, J. C. Lennox, B. H. Neumann, H. Smith and J. Wiegold, 'Groups with all subgroups normal-by-finite', J. Aust. Math. Soc. Ser. A 59 (1995), 384-398.

[4] C. Casolo, 'Groups with finite conjugacy classes of subnormal subgroups', Rend. Semin. Mat. Univ. Padova 81 (1989), 107-149.

[5] M. R. Dixon, Sylow Theory, Formations and Fitting Classes in Locally Finite Groups (World Scientific, Singapore, 1994).

[6] M. R. Dixon, M. J. Evans and H. Smith, 'Some countably recognizable classes of groups', J. Group Theory 10 (2007), 641-653.

[7] F. de Giovanni and C. Rainone, 'Infinite groups with many generalized normal subgroups', Internat. J. Group Theory 1 (2012), 39-49.

[8] W. Möhres, 'Auflösbarkeit von Gruppen, deren Untergruppen alle subnormal sind', Arch. Math. (Basel) 54 (1990), 232-235.

[9] B. H. Neumann, 'Groups with finite classes of conjugate subgroups', Math. Z. 63 (1955), 76-96.

[10] D. J. S. Robinson, Finiteness Conditions and Generalized Soluble Groups (Springer, Berlin, 1972).

F. DE GIOVANNI, Dipartimento di Matematica e Applicazioni,

Università di Napoli Federico II, Complesso Universitario Monte S. Angelo,

Via Cintia, I - 80126, Napoli, Italy

e-mail: degiovan@unina.it

M. MARTUSCIELLO, Dipartimento di Matematica e Applicazioni,

Università di Napoli Federico II, Complesso Universitario Monte S. Angelo,

Via Cintia, I - 80126, Napoli, Italy

e-mail:maria.martusciello@unina.it

C. RAINONE, Dipartimento di Matematica e Applicazioni,

Università di Napoli Federico II, Complesso Universitario Monte S. Angelo,

Via Cintia, I - 80126, Napoli, Italy

e-mail: caterina.rainone@unina.it 